\title{
Eating following cholinergic stimulation of the hypothalamus
}

\author{
WILLIAM T. CHANCE \\ Department of Pharmacology, Medical College of Virginia, Richmond, Virginia 23298
}

and

CARLTON E. LINTS

Northern Illinois University, DeKalb, Illinois 60115

\begin{abstract}
Stimulation of the perifornical hypothalamus of satiated rats with both crystalline carbachol and solutions of the drug elicited eating and drinking. The eating response did not appear to be a rebound phenomenon, since it paralleled the elicitation of drinking and was not inhibited by repeated applications of crystalline carbachol at 20-min intervals. Eating following the injection of various doses $(0.5,1.0,2.0$, and 4.0 nanomoles) of carbachol also paralleled drinking, showing appropriate dose-response relationships. It is hypothesized that cholinergically elicited eating may involved excitatory interaction with adrenergic systems, with increased nicotinic cholinergic activity stimulating alpha-adrenergic neurons to elicit eating. Alternatively, cholinergic stimulation may be activating adrenergic systems through a direct presynaptic release of norepinephrine or epinephrine.
\end{abstract}

Past research has suggested CNS neurochemical specificity in the control of food and water intake. Thus, intrahypothalamic application of the cholinomimetic drug, carbachol, elicited drinking and inhibited eating, while administration of norepinephrine to the same anatomical locus elicited eating and inhibited drinking (Grossman, 1962). These observations have been extended by demonstrations of mutual reciprocal antagonism between adrenergic and cholinergic systems in the mediation of consummatory behavior (Singer \& Kelly, 1972). Thus, norepinephrine effectively antagonized drinking elicited by carbachol, water deprivation, or hypertonic saline. Conversely, carbachol reduced eating following the injection of norepinephrine or food deprivation.

Although these experiments suggest a degree of neurochemical specificity, several reports of eating following cholinergic manipulations have been largely ignored. A cholinergic link in the mediation of eating has been suggested by experiments using electrical stimulation of hypothalamic nuclei to elicit or inhibit food intake. In these studies, systemic injection of the anticholinesterase compound, physostigmine, significantly reduced the current threshold required in the lateral area to elicit eating (Stark, Totty, Turk, \& Henderson, 1968). Furthermore, systemic injection of physostigmine increased the current threshold to inhibit eating in the ventromedial area of food-deprived rats (Stark, Turk, \& Totty, 1971). Both of the above effects could also be

This research was supported, in part, by NIH-DA-00296-03. blocked by pretreatment with the antimuscarinic drug, atropine. Therefore, these reports suggest that the probability of eating following electrical stimulation of the hypothalamus is increased when the break-down of endogenous acetylcholine is inhibited.

Direct application of carbachol to the hypothalamus has been observed to elicit eating in satiated rats, while atropine inhibited eating in food-deprived rats (Capobianco \& Mountford, 1974). Injection of carbachol into the lateral and preoptic hypothalamus of the rabbit has also been reported to elicit eating in a dose-dependent manner (Sommer, Novin, \& Le Vine, 1967). In addition, investigators have observed eating as well as other nonspecific behavioral effects following cholinergic stimulation of the rat lateral hypothalamus (Coury, 1967; Myers, 1964). In systematic investigations of the specificity of behaviors elicited by chemical stimulation of the hypothalamus, perifornical hypothalamic (PFH) application of crystalline carbachol elicited eating in female hooded rats (Chance, 1972). Furthermore, eating was elicited at hypothalamic sites $(4.4 \mathrm{~mm}$ anterior to the interaural line) not affected by norepinephrine. In a subsequent experiment (Chance, Note 1), carbachol and norepinephrine elicited comparable food intake at PFH sites $1 \mathrm{~mm}$ anterior to the loci of the previous study.

Wise (1972) has also reported eating following application of crystalline carbachol to the hypothalamus. He did not attribute this eating response to a direct effect of the drug, however, but interpreted it to be a rebound phenomenon. Thus, the rebound hypothesis maintains that cholinergic stimu- 
lation is inhibitory to eating, which is mediated by adrenergic neurochemical systems. Therefore, eating following the application of carbachol results when this inhibition is terminated, resulting in excitatory adrenergic activity. Thus the disinhibition of adrenergic systems is postulated to elicit eating following cholinergic stimulation of the hypothalamus.

\section{EXPERIMENT 1}

If eating elicited by the administration of carbachol is a rebound phenomenon, one would expect that drinking would occur before eating on any given trial and eating should be inhibited during that part of the trial when drinking is observed. Furthermore, restimulation of the animal with carbachol at the appropriate time should maintain the inhibition and prevent the occurrence of rebound eating. This experiment was designed to test these hypotheses using crystalline carbachol to elicit eating and drinking in satiated animals.

\section{Method}

Subjects. Eight adult, male, Long-Evans rats served as subjects in this experiment. They were individually housed in a temperature controlled environment under a 12-h light/dark cycle and were maintained ad lib on ground rat chow and water.

Apparatus. Double cannulae (22 ga/30 ga) were constructed from Yale Stainless steel hypodermic needles and implanted using a Model $51200 \mathrm{Krieg}$ stereotaxic instrument. All testing was conducted in the home cages $(28 \times 22 \times 20 \mathrm{~cm})$, which had wire fronts allowing easy observation of the rats. Ground rat chow was provided in nonscatter food cups located at the front of the cages adjacent to the water bottles. All determinations of food and water consumption were made using a Mettler P-1200 balance. accurate to the nearest hundredth gram.

Procedure. The subjects were anesthetized with Nembutal (40 mg/kg IP; Abbott Labs, Chicago), and the outer cannulae (22 ga) were implanted unilaterally into the PFH at the following coordinates: anterior $(A)=6.4$, lateral $(L)=1.0$, and ventral $(V)=-2.0 \mathrm{~mm}$ (Pellegrino \& Cushman, 1967). The rats were allowed at least 1 week to recover from surgery before any testing was initiated.

In order to allow the subjects to serve as their own controls, a trial consisted of two stimulation periods of $60 \mathrm{~min}$ duration each. During the first period (sham stimulation control), no drugs were administered. Each subject was removed from the home cage and weighed; then the empty inner cannula ( $30 \mathrm{ga})$ was inserted. The rat was immediately returned to the cage, and food and water intake were determined at the end of the period. The drug stimulation period followed immediately and was initiated by the insertion of the drug-containing cannulae. With the aid of a dissecting microscope, each inner cannula was tapped three times into a small amount of crystalline carbamylcholine (Sigma Chemical Co., St. Louis). Although accurate specification of dose using crystalline stimulation techniques is impossible, five taps of a 30-ga needle into a pile of carbachol crystals have been reported to transfer approximately $2.8 \mu \mathrm{g}$ (range $=1.6$ to $6.0 \mu \mathrm{g}$ ) of the drug (Levitt, White, \& Sander, 1970). At the end of the 60-min drug-stimulation period, food and water intake were again determined.

The first 4 days of this experiment consisted of daily sham stimulation followed by drug-stimulation periods. During the periods of carbachol stimulation, the behavior of each subject was monitored each minute by an observer for the occurrence of eating and/or drinking. These observations were made to evaluate the relationship between eating and drinking as well as their respective latencies. On the fifth trial, the subjects were sham stimulated as usual, but prior to the drug stimulation they were randomly divided into two groups of four rats each. In order to assess the effeci of repeated applications of carbachol on eating, Group 1 received three carbachol stimulations equally spaced across the $60-\mathrm{min}$ period (one initially and one at 20 and $40 \mathrm{~min}$ thereafter). Group 2 was initially stimulated with carbachol and then sham stimulated (by removing each rat from the cage and removing and reinserting the inner cannula) at 20-min intervals. As in the preceding days of the experiment, the eating and drinking responses of each subject were recorded for each minute of the period. Food and water consumption were also measured for each period of stimulation in this experiment.

Statistical analyses of the data were accomplished using a repeated measures analysis of variance (ANOVA) with individual comparisons made by $t$ tests. Correlative relationships between eating and drinking were based on the Pearson product moment coefficient of correlation. At the conclusion of the experiment, all subjects were sacrificed by decapitation, the brains were removed and stored in Formalin $(10 \%)$, and frozen $40-\mu$ sections were directly projected onto photographic paper to reveal cannulae placements.

\section{Results and Discussion}

Mean food and water intake during the sham and drug stimulation periods is presented in Figure 1. As in previous experiments (Chance, 1972, Note 1), there were significant increases in both eating $[\mathrm{F}(1,21)$ $=26.53, \mathrm{p}<.01]$ and drinking $[\mathrm{F}(1,21)=56.01$, $p<.01$ ] across the first four trials following the administration of carbachol. Although the drug effect was depressed on the first trial, both food and water intake reached asymptote following the second

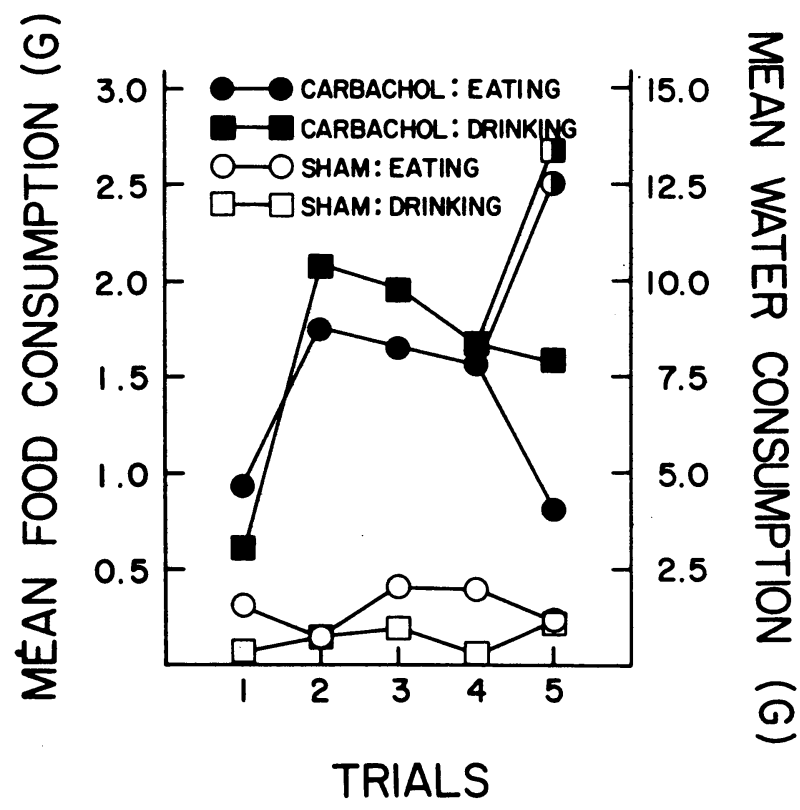

Figure 1. Mean food and water consumption following the daily administration of crystalline carbachol or the preceding sham stimulation of the eight rats in Experiment 1. On Trial 5 the rats randomly divided into two groups of four each. Group 1 (O) received three carbachol stimulations ( $20 \mathrm{~min}$ apart), while Group $2(\bullet)$ was initially given carbachol followed by two sham stimulations at 20 -min intervals. 
application of carbachol. When the three stimulations with carbachol were administered (Trial 5), no inhibition of eating or drinking was observed. In fact, restimulation elicited significantly increased food $[\mathrm{t}(6)=3.03, \mathrm{p}<.05]$ and water $[\mathrm{t}(6)=2.23$, $\mathrm{p}<.05$; one-tailed $\mathrm{t}$ ] intake. Contrary to what is predicted by the rebound hypothesis, no inhibition of eating was observed following the repeated applications of carbachol. The significant increase in eating and drinking following these stimulations suggests that both behaviors are primary effects of the drug.

The mean percentage of subjects eating and drinking over the five drug stimulation periods is presented in Figure 2. These data are grouped into 5-min intervals to reveal the pattern of development and relationship of the two behaviors. Across the first two trials, there were negative correlations between the occurrence of eating and drinking $(r=-0.10$, Trial 1, Panel A; $r=-0.51$, Trial 2, Panel B). During Trials 3 (Panel C) and 4 (Panel D), however, the correlations were significant in the positive direction ( $r=0.70$ and 0.53 , respectively). Panels $E$ and $F$ illustrate the patterns of eating and drinking for Trial 5, when the three carbachol (Panel E) or sham (Panel F) stimulations were administered. At no time did restimulation with carbachol elicit a decreased response. Instead, the eating responses tended to increase with each additional stimulation.

These patterns of eating and drinking following cholinergic stimulation of the PFH suggest a biphasic response. Thus, with the first two applications of the drug, eating occurred predominantly in the last half of the period and was negatively correlated with drinking. On the third and fourth trials, however, eating and drinking both occurred primarily in the first $35 \mathrm{~min}$ of the period, and there were significant positive correlations between the two behaviors. When the three carbachol stimulations were administered (Trial 5), eating was maintained and increased following each stimulation, while the additional sham stimulations effectively terminated the behavior. Therefore, carbachol-elicited eating may appear initially to be a rebound phenomenon as reported (Wise, 1972). Following several daily stimulations, however, both eating and drinking are elicited shortly after administration of the drug. In previous research (Chance, 1972, Note 1), daily applications of the drug were necessary to maintain the eating response. If no stimulations were given for several days, the eating effect was diminished and gradually incremented with resumption of drug treatment. Thus, it is possible for one laboratory to report inhibition of eating (Grossman, 1962), while others observe facilitation of eating in satiated animals following administration of the drug.

Histological examination indicated that the cannulae created tracts approximately $1.0 \mathrm{~mm}$ in diameter. These tracts were centered at approximately $5.5 \mathrm{~mm}$ anterior to the interaural line, $1.0 \mathrm{~mm}$ lateral to the third ventricle, and terminated at $0.8 \mathrm{~mm}$ above the base of the brain.
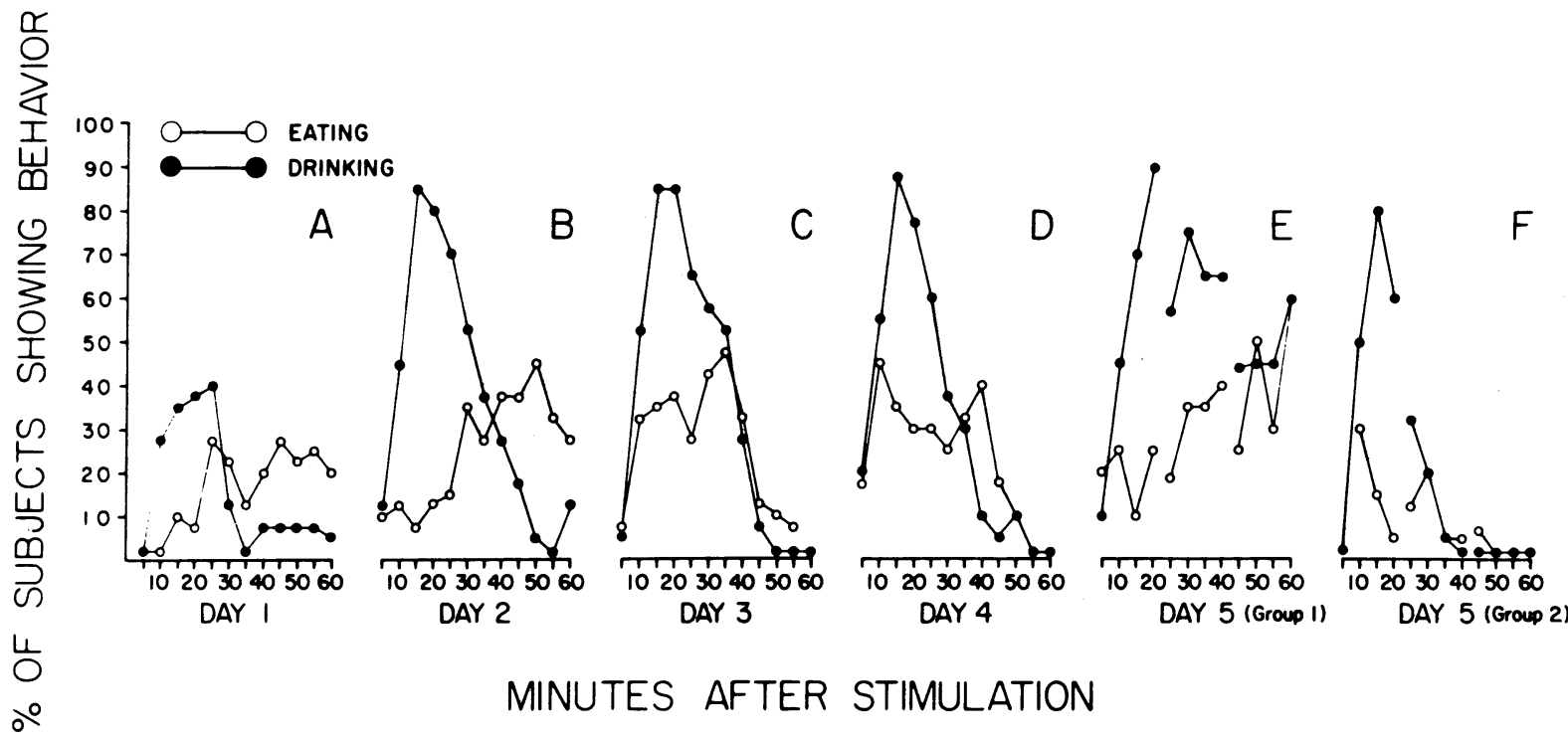

\section{MINUTES AFTER STIMULATION}

Figure 2. Patterns of eating and drinking during the $60 \mathrm{~min}$ following the administration of carbachol to the eight rats in Experiment 3. The data are grouped into blocks of means across 5-min periods. On Day 5, Group 1 (Panel E) received three stimulations with carbachol at 20-min intervals, while Group 2 (Panel F) was initially stimulated with the drug and sham stimulated at 20 and $\mathbf{4 0} \mathrm{min}$ afterward. 


\section{EXPERIMENT 2}

Although the previous experiment suggested that eating may be a primary effect of cholinergic stimulation, the use of crystalline stimulation techniques precluded dose-response comparisons. If eating following cholinergic stimulation of the hypothalamus is reflective of physiological mechanisms, it should be elicited by injection of solutions of the drug. Furthermore, the eating response should exhibit appropriate dose-response relationships and parallel the elicitation of drinking. Therefore, it was the purpose of this experiment to investigate eating and drinking elicited by various doses of carbachol in solutions.

\section{Method}

Subjects. Forty-nine adult, male, Sprague-Dawley rats served as subjects in this experiment. These animals were maintained under identical environmental conditions as those subjects of the first experiment.

Apparatus. The apparatus were maintained as in Experiment 1, except that single cannulae ( $24 \mathrm{ga})$ were constructed from the hypodermic needles. To prevent occlusion, 31-ga stylets obturated the implanted cannulae between periods of stimulation. All injections were administered in $1-\mu \mathrm{l}$ volumes and delivered through a 31-ga hypodermic needle, cut to terminate flush with the cannulae. The injection volume was measured with a Hamilton $50-\mu$ l syringe $(705-\mathrm{LT})$ and controlled using a Hamilton repeating dispenser (PB-600-1).

Procedure. As in the preceding experiment, the subjects were anesthetized and the cannulae were implanted unilaterally into the PFH $(\mathrm{A}=6.5, \mathrm{~L}=1.0$, and $\mathrm{V}=-2.0 \mathrm{~mm}$; Pellegrino \& Cushman, 1967). After recovering from surgery, the rats were randomly assigned to five groups. Again, a within-subjects design allowed all rats to serve as their own controls, by assessing eating and drinking following $(60 \mathrm{~min})$ the injection $(1 \mu \mathrm{l})$ of normal saline. In addition, immediately after the termination of the initial control period, the administration of $1 \mu \mathrm{l}$ of saline to one group $(n=10)$ or different doses of carbachol across the other groups allowed between-subjects comparisons. Eating and drinking following $(60 \mathrm{~min})$ the administration $(1 \mu \mathrm{l})$ of $0.5(\mathrm{n}=11)$, $1.0(\mathrm{n}=11), 2.0(\mathrm{n}=8)$ and $4.0(\mathrm{n}=9)$ nanomoles (nmol) of carbachol (free base) was assessed across five daily trials. As in the previous experiment, these tests were conducted in the home cages during the morning hours, when the rats should be experiencing maximum satiation.

The data were evaluated using a repeated-measures ANOVA, and the animals were sacrificed for histological verification of cannulae placements at the close of the experiment.

\section{Results and Discussion}

Histological examination revealed that the average cannula tract $(0.7 \mathrm{~mm}$ in diameter) was well localized in the PFH at the level of the tuber cinereum. The tracts terminated at approximately $1.6 \mathrm{~mm}$ above the base of the brain, $1.2 \mathrm{~mm}$ lateral to the third ventricle, $0.3 \mathrm{~mm}$ from the fornix, and $5.3 \mathrm{~mm}$ anterior to the interaural line.

Mean food and water intake following the administration of saline and the various doses of carbachol is presented in Figure 3. Each point is based on the response during the second 60-min period and is averaged across the five trials. Both food $[F(4,44)=22.59, \mathrm{p}<.01]$ and water $[\mathrm{F}(4,44)=$

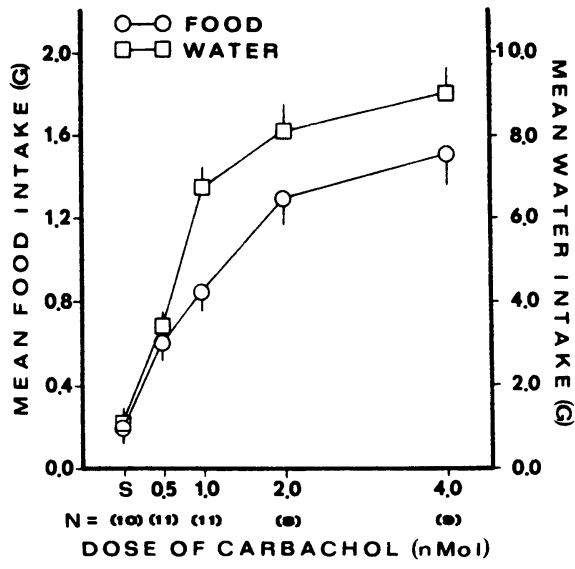

Figure 3. Mean ( \pm S.E.M.) food and water intake following the injection of saline and various doses of carbachol. Each point represents the mean of the five trials of Experiment 2.

$28.54, \mathrm{p}<.01]$ intake increased with increasing doses of carbachol. Although the magnitude of both responses was similar to that observed following crystalline stimulation, neither behavior incremented across trials. This difference between techniques may reflect differences in diffusion of the drug to effective sites.

Each dose of carbachol elicited significantly more eating and drinking across the five trials than did the second injection of saline in the control group. Comparison of within data (each subject as own control), however, indicated that only the two highest doses of carbachol elicited more eating, while each of the doses elicited more drinking than did the preceding control injection of normal saline.

A similar dose-response analysis of carbacholelicited drinking has been reported (Miller, Gottesman, \& Emery, 1964). However, these investigators observed considerably less drinking, at their lower dose values, than reported in the present study. They also report that eating was not reliably enhanced by the carbachol treatments. These differences may be reconciled by methodological differences in the two studies, however, since Miller et al. (1964) did not test in the home cages, probably stimulated a less effective area, and selected their subjects on the basis of prior drinking tests. As previously indicated (Chance, 1972; Wise, 1972; Chance, Note 1), carbachol-elicited eating is not a robust effect and may be particularly subject to changes in environmental variables. Thus, precise control of environmental conditions will facilitate the accurate measurement of this reliable effect.

In this second experiment, both eating and drinking were elicited by all doses of carbachol. Both of these behaviors increased with increasing doses of the drug, indicating appropriate dose-response relationships. Although minute-by-minute observation of the animals was not performed, as in Experiment 1, 
informal observation suggested that the eating response to liquid injections had a longer latency than that noted following crystalline stimulation. The animals typically ate after a $10-$ or 20 -min latency, while the latency observed on the fourth crystalline stimulation day was approximately 5 to $10 \mathrm{~min}$. Conversely, the animals drank within 1 to 3 min after the liquid injections, while the latency to drink using crystalline techniques was typically 5 to $10 \mathrm{~min}$.

\section{GENERAL DISCUSSION}

These experiments suggest that eating following cholinergic stimulation of the PFH is not a rebound phenomenon or an artifact or crystalline stimulation techniques. Although of longer latency than the drinking response, carbachol-induced eating appears to be a direct consequence of the drug. Several reports (Grossman, 1962; Singer \& Kelly, 1972) have indicated that carbachol is inhibitory to eating. However, more recent data (Capobianco \& Mountford, 1974) have shown differential effects of cholinergic agonists and antagonists, depending upon the degree of satiation. Using liquid injections of carbachol $(4.0 \mathrm{nmol})$, we have observed stimulation-induced reduction of eating in food-deprived rats (unpublished observations) and suggest that this effect may be interpreted on the basis of drive competition. Since carbachol apparently makes the animal thirsty, more time in the measurement period will be spent drinking. This interpretation is particularly attractive when one realizes that the typical reduction of eating following administraton of carbachol to fooddeprived rats is only $20 \%$ to $25 \%$ (Singer \& Kelly, 1972; Chance, unpublished observations).

Since most of the research has indicated adrenergic systems as mediators of food intake (Grossman, 1962; Leibowitz, 1970; Miller et al., 1964; Slangen \& Miller, 1969), one may postulate that cholinergically induced eating may occur through a cholinergicadrenergic interaction within the hypothalamus. A model for this type of interaction exists within the peripheral autonomic nervous system, with preganglionic nicotinic activity of acetycholine eliciting the release of norepinephrine at postganglionic sympathetic sites. Since the excitatory adrenergic control of food intake has been reported to be due to alphaadrenergic activity (Leibowitz, 1970), testable hypotheses of this interaction may be generated. Thus, pretreatment with either a nicotinic antagonist or alpha-adrenergic antagonist are hypothesized to block carbachol-elicited eating. Similar tests have previously shown the drinking response to cholinergic stimulation to be due to the muscarinic actions of carbachol (Levitt \& Fisher, 1966).

An alternative model to explain eating following cholinergic stimulation may be that adrenergic neurons require the release of acetylcholine to initiate adrenergic activity (Burn \& Rand, 1960). This model could also be tested pharmacologically, by using drugs that antagonize the release of acetylcholine or deplete adrenergic stores.

\section{REFERENCE NOTE}

1. Chance, W. T. Drinking and eating elicited by direct cholinergic stimulation of the lateral hypothalamus of the rat. Paper presented at the meeting of the Midwestern Psychological Association. Chicago, May 1973.

\section{REFERENCES}

BuRN, J. H.. \& RAND, M. J. Sympathetic postganglionic cholinergic fibres. British Journal of Pharmacology, 1960, 15, 55-66.

CHANCE. W. T. A test of the chemical specificity of neural circuits subserving drinking in the lateral hypothalamus of the adult female hooded rat. Unpublished masters thesis, Northern Illinois University, 1972.

Capobianco, S., \& Mountford, D. The effects of drug administration to the lateral hypothalamus: Neurochemical coding or nonspecificity? Bulletin of the Psychonomic Society, 1974, 3, 179-180.

Coury, J. N. Neural correlates of food and water intake in the rat. Science. 1967, 156, 1763-1765.

Grossman, S. P. Direct adrenergic and cholinergic stimulation of hypothalamic mechanisms. American Journal of Physiology, 1962. 202, 872-882.

Leibowitz, S. F. Hypothalamic beta-adrenergic "satiety" system antagonizes an alpha-adrenergic "hunger" system in the rat. Nature, 1970, 226, 963-964.

LeVitT, R. A., \& Fisher, A. E. Anticholinergic blockade of centrally induced thirst. Science, 1966, 154, 520-522.

LevitT, R. A., White, C. S., \& SANDER, D. M. Dose-response analysis of carbachol-elicited drinking in the rat limbic system. Journal of Comparative and Physiological Psychology, 1970. 72, 345-350.

Miller, N. E., Gottesman, K. S., \& Emery, N. Dose response to carbachol and norepinephrine in rat hypothalamus. American Journal of Physiology, 1964, 206, 1384-1388.

Myers, R. D. Modification of drinking patterns by chronic intracranial chemical infusion. In M. J. Wayner (Ed.), Thirst in the regulation of body water. Oxford: Pergamon Press, 1964. Pp. 533.549.

Pellegrino, L. J., \& Cushman, A. J. A stereotaxic atlas of the rat brain. New York: Appleton-Century-Crofts, 1967.

Singer, G.. \& Kelly, J. Cholinergic and adrenergic interaction in the hypothalamic control of drinking and eating behavior. Phys. iology and Behavior, 1972, 8, 885-890.

Slangen, J. L., \& Miller, N. E. Pharmacological tests for the function of hypothalamic norepinephrine in eating behavior. Physiology and Behavior, 1969, 4, 543-552.

Sommer, J. L., Novin, D., \& LE VINE, M. Food and water intake after intrahypothalamic injections of carbachol in the rabbit. Science, 1967, 156, 983-984.

Stark, P.. Turk, J. A., \& Totty, C. W. Reciprocal adrenergic and cholinergic control of hypothalamic-elicited eating and satiety. American Journal of Physiology, 1971, 5, 1516-1521.

Stark, P., Totty, C. W., Turk, J. A., \& Henderson, J. K. A possible role of a cholinergic system affecting hypothalamic-elicited eating. American Journal of Physiology, 1968, 214, 463-468.

WISE, R. A. Rebound eating following carbachol-induced drinking in rats. Physiology and Behavior, 1972, 9, 659-661.

(Received for publication July 5, 1977; revision accepted September 2, 1977.) 